\title{
Interest of Sclerosing Injections in Hemorrhoidal Pathology: Experience of the Service of Hepato-Gastroenterology of Fez
}

\author{
H. Abid ${ }^{1,2}$, R. Benjira ${ }^{1,2}$, M. Lahlali ${ }^{1,2}$, A. Lamine ${ }^{1,2}$, N. Lahmidani ${ }^{1,2}$, M. Elyousfi ${ }^{1,2}$, N. Aqodad ${ }^{1,2}$, \\ D. Benajah", ${ }^{1,2}$ A. Ibrahimi1,2, M. Elabkari1,2
}

${ }^{1}$ Hepato-Gastroenterology Department, Hassan II University Medical Center, Fez, Morocco

${ }^{2}$ Faculty of Medicine, Sidi Mohammed Ben Abdellah University, Fez, Morocco

Email: abidhakima@hotmail.com

How to cite this paper: Abid, H., Benjira, R., Lahlali, M., Lamine, A., Lahmidani, N., Elyousfi, M., Aqodad, N., Benajah, D., Ibrahimi, A. and Elabkari, M. (2019) Interest of Sclerosing Injections in Hemorrhoidal Pathology: Experience of the Service of Hepato-Gastroenterology of Fez. Open Journal of Gastroenterology, 9, 36-42. https://doi.org/10.4236/ojgas.2019.91006

Received: December 14, 2018

Accepted: January 21, 2019

Published: January 24, 2019

Copyright $\odot 2019$ by author(s) and Scientific Research Publishing Inc. This work is licensed under the Creative Commons Attribution International License (CC BY 4.0).

http://creativecommons.org/licenses/by/4.0/

\begin{abstract}
Hemorrhoids are a clinical and anatomical subject that has not been completely investigated [1]. This benign condition is expressed by intermittent functional manifestations as rectorrhagies and prolapse. While the medical treatments seek to relieve symptoms, the instrumental ones are intended to reposition and hold the hemorrhoids in an anatomically correct position and to reduce their vascularity [1]. Indeed, sclerotherapy seems an effective and safe method in fact. Aim of the Study: To report the results of a moroccan series of patients treated with sclerosing injections, emphasizing short- and long-term efficacy as well as the rate of complications. Methods: This is a descriptive retrospective study of the 148 patients with symptomatic internal hemorrhoids who have been treated with sclerotherapy in the gastroenterology department of the university medical center of Fez over a period of 17 years [2001-2018]. Results: The average age of our patients is 47.1 years [19 - 86]. We notified a large male predominance with a sex ratio M/F 2.58. Rectorrhagies and prolapse were the most frequent reasons for consultation; they were respectively notified in $96 \%(\mathrm{n}=143)$ and $83 \%$ of patients $(\mathrm{n}=$ 123). Anemia was found in 45 cases (30.4\%), of which 29 cases required transfusion. Proctologic examination found internal hemorrhoids grade 2 in $83 \%(\mathrm{n}=124)$ and grade 1 in $16.2 \%(\mathrm{n}=24)$ of patients. Initial success was found in 119 cases $(80.4 \%)$ after an average of 2.25 sessions $(1-4)$. Nine patients $(8,11 \%)$ had minor complications dominated by minimal rectorrhagies in 8 cases, resolved spontaneously. In 29 cases (19.5\%), the sessions were interrupted following a failure of the technique in 12 cases (41.3\%), in 11 cases after change of the stage of hemorrhoids of stage II to III, in 4 cases following the appearance of an anal fissure contraindicating the
\end{abstract}


procedure, and in 2 cases due to severe pain post sclerosis. Among these 29 cases, 13 patients were referred for surgery, while in 16 patients we opted for an instrumental treatment by ligature. The initial evolution was specified with a average follow-up of 3 months ( 1 - 13 months). The recurrence rate was 22.3\% (N: 33 cases) after an average follow-up of 9.5 months (1 - 48 months). In the long term (over one year) and in the short term, the recidivism rate was $6 \%(\mathrm{n}=9$ cases $)$ and $16.2 \%(\mathrm{n}=24)$ respectively. Among recurrent patients, the sclerosis protocol was repeated in 20 patients $(60.6 \%)$ with a success rate of $85 \%$; for the remaining 13 patients, we opted for elastic ligation in 4 patients, and for surgery in 6 patients, while 3 patients were lost to follow-up. Conclusion: Since the end of the XIX century, sclerosis has been an instrumental technique, simple, inexpensive, and effective for the treatment of symptomatic internal hemorrhoids of grades 1 and 2, allowing good results in the long run, with minimal risk of complications.

\section{Keywords}

Internal Hemorrhoids, Sclerosing Injection, Evolution, Complications

\section{Introduction}

Hemorrhoids are a clinical and anatomical subject that has not been completely investigated [1]. This benign condition is expressed by intermittent functional manifestations as rectorrhagies and prolapse. while the medical treatments seek to relieve symptoms, the instrumental ones are intended to reposition and hold the hemorrhoids in an anatomically correct position and to reduce their vascularity [1].

Sclerotherapy is an effective technique widely used around the world that aims to create a cicatricial fibrosis which fixes the mucosa in the deep plane and reduces the vascularization, thus allowing the symptomatic relief (rectorrhagies) and the correction of a possible anatomical defect (prolapse).

\section{Goal of the Study}

Our goal is to present a moroccan series of patients with symptomatic internal hemorrhoids treated with sclerosing injections, it is a series of 148 patients collected in the department of hepatogastroenterology of the university medical center Hassan II of Fez. Through this series, we seek to analyze the epidemiological characteristics of these patients, to report our experience concerning sclerotherapy and its rate of complications as well as comparing our results with those of the international literature.

\section{Materials and Methods}

This is a descriptive retrospective study of patients with symptomatic internal hemorrhoids treated by sclerotherapy in ambulatory digestive functional 
exploration unit of the hepatogastroenterology department of the university medical center Hassan II of Fez between April 2001 and April 2018. All patients lost to follow-up and those refusing to complete the protocol fixed at 4 sessions were excluded.

The variables were retrospectively collected in a computer database then their statistical analysis was performed using SPSS 20.0.

There were no limitations of this study.

\subsection{Technique}

The sclerosing product used is the double hydrochloride of Quinine and urea (Kineara).

It is injected with an extension and a short needle in the submucosa (Figure 1). The operator must dislocate the anoscope in order to obtainin the light of the anoscope a mucous crescent sushemorrhoidal.

Before the injection of liquid, the needle mustbe mobilized slightly because if it is fixed in the muscular, the injection will be painful and ineffective.

If the injection is too superficial,it creates an edema in "Fish bladder", giving a painful impression foreign intra-anal body.

At each session, the volume injected at the opposite poles is 2 to $5 \mathrm{~mL}$ and avoiding injection at the anterior pole (rectovaginal septum or lodgeprostatic) and posterior pole (anococcygeal raphe) [1] [2].

\subsection{Contraindications to Quinine Hydrochloride Urea [2]}

- The pregnancy;

- Optic neuritis;

- Anticoagulant treatments.

Recurrence: Sessions can be repeated two to four timeswith 21-day therapeutic windows.

Indications: bleeding in case of internal hemorrhoids grades 1 and 2 after failure of medical treatment (2).

Dietary and defecatory hygiene measures were prescribed to all patients. A transit regulator has been prescribed for subjects suffering from constipation. All our patients were informed about the indication of sclerosis, the course of the

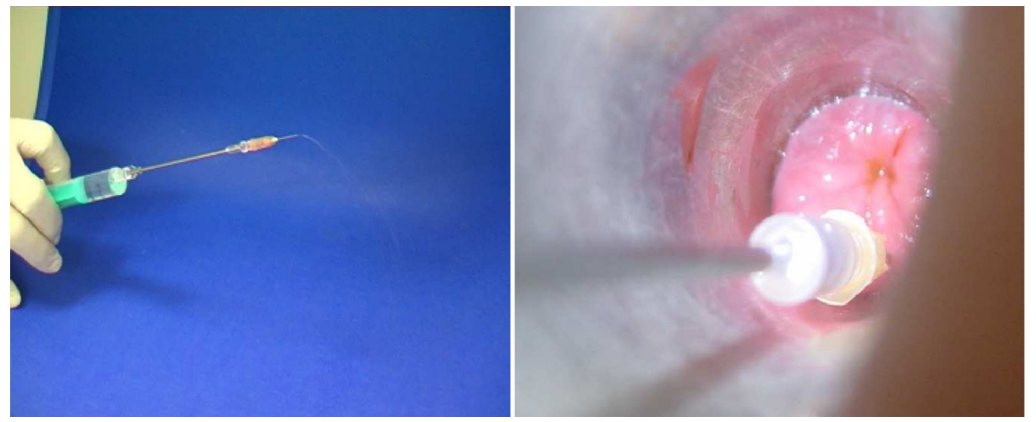

Figure 1. Material of sclerosis. 
technique, the incidents and possible complications. All our patients received post-sclerosis antibiotics (metronidazole $1.5 \mathrm{~g}$ daily) combined with analgesics for 72 hours.

The therapeutic objective was the disappearance of clinical symptomatology after 1 to 4 sessions of sclerosis spaced at 3 to 4 weeks.

\subsection{Defintions}

- Short term success. the disappearance of the symptomatology after one to four sessions.

- Long term succes. non reappearance of symptoms more than one year after the end of sclerosis protocol.

- Failure: the persistence of symptoms after a maximum of 4 sessions.

- Short-term recurrence rate: the reappearance of symptoms less than one year after the end of the sclerosis protocol. It has been clarified, of course, only in patients who have successfully responded to the sclerosis protocol.

- Long-term recurrence rate: as the reappearance of symptoms more than one year after the end of the sclerosis protocol.

\section{Results}

During the study period, treatment of internal hemorrhoids with sclerotherapy wasindicated in 148 patients. Their mean age was 47.1 years [19 - 86 years]. A clear male predominance was observed with a sex ratio M/F 2, 52. Rectorrhagies and prolapse were the most common reasons for consultation; they were notified in ( $\mathrm{n}=143$ and 123 patients respectively. Anemia was found in 45 cases (30.4\%) of which 29 cases (64\%) required transfusion (Table 1).

Initial success was found in 119 cases (80\%) after an average of 2.25 sessions [1] [3]. Twelve patients (8.1\%) had minor complications dominated by minimal rectorrhagies in 8 cases resolved spontaneously. In 29 cases (13.51\%), the sessions were interrupted: following a failure of the technique in 12 cases (41.3\%), in 11 cases after change of the stage of hemorrhoids of stage II to III, in

Table 1. Clinical characteristics of the enrolled patients.

\begin{tabular}{lc}
\hline & Patients $(\mathbf{n}=148)$ \\
\hline Mean age & 47.1 years [19-86 years] \\
Sex & $106(71.6 \%)$ \\
Male & $42(28.3 \%)$ \\
Female & \\
Symptoms & $143(96.6 \%)$ \\
Rectorrhagies & $123(83 \%)$ \\
Prolapse & $46(31 \%)$ \\
Constipation & $26(17.5)$ \\
Proctalgia & \\
Internal hemorrhoids grade & $24(16.2 \%)$ \\
Grade I & $124(83 \%)$ \\
Grade II & \\
\hline
\end{tabular}


4 cases following the appearance of an anal fissure contra-indicating the gesture, and in 2 cases due to severe pain post sclerosis. Of these 29 cases, 13 patients were referred to surgery, while in 16 patients we opted for instrumental ligation. The initial evolution was specified with a mean follow-up of 3 months (1 - 13 months). The recurrence rate was $22.3 \%$ ( $\mathrm{n}=33$ cases) after an average follow-up of 9.5 months ( 1 - 48 months). In the long term (over one year) it was notified in 9 patients $(6 \%)$, while it wasobserved in 24 cases $(16.2 \%)$ in the short term. Among recurrent patients, the sclerosis protocol was resumed in 20 patients with a success rate of $85 \%$; for the remaining 13 patients, we opted for elastic ligation in 4 patients, and for surgery in 6 patients, while 3 patients were lost to follow-up (Figure 2).

\section{Discussion}

The objective of the instrumental treatment is to create a cicatricial fibrosis which fixes the mucosa in the deep plane and reduces the vascularization thus allowing the symptomatic relief (Rectorrhagies) and the correction of a possible anatomical defect (prolapse). These methods apply a chemical or physical process to treat internal hemorrhoidal disease [1].

The short-term efficacy of sclerosis is identical to that observed for elastic ligation or infrared photocoagulation [3] [4] [5]. In the long term and especially at three years, the efficacy of sclerosis is comparable to photo-coagulation's one but lower than that observed for elastic ligation with a benefit always present in $70 \%$ to $90 \%$ of cases (haemorrhagic hemorrhoids not prolapsed or moderate prolapse). Indeed, if $25 \%$ of patients treated by sclerosis are asymptomatic at 5 years, the benefit in patients treated by elastic ligation is $75 \%$ to $90 \%$.

For the treatment of prolapse, sclerotherapy seems much less effective than elastic ligation [5] [6]. Patients treated with elastic ligation had fewer recourse to other techniques than those treated with infrared or sclerosing injection [7]. In

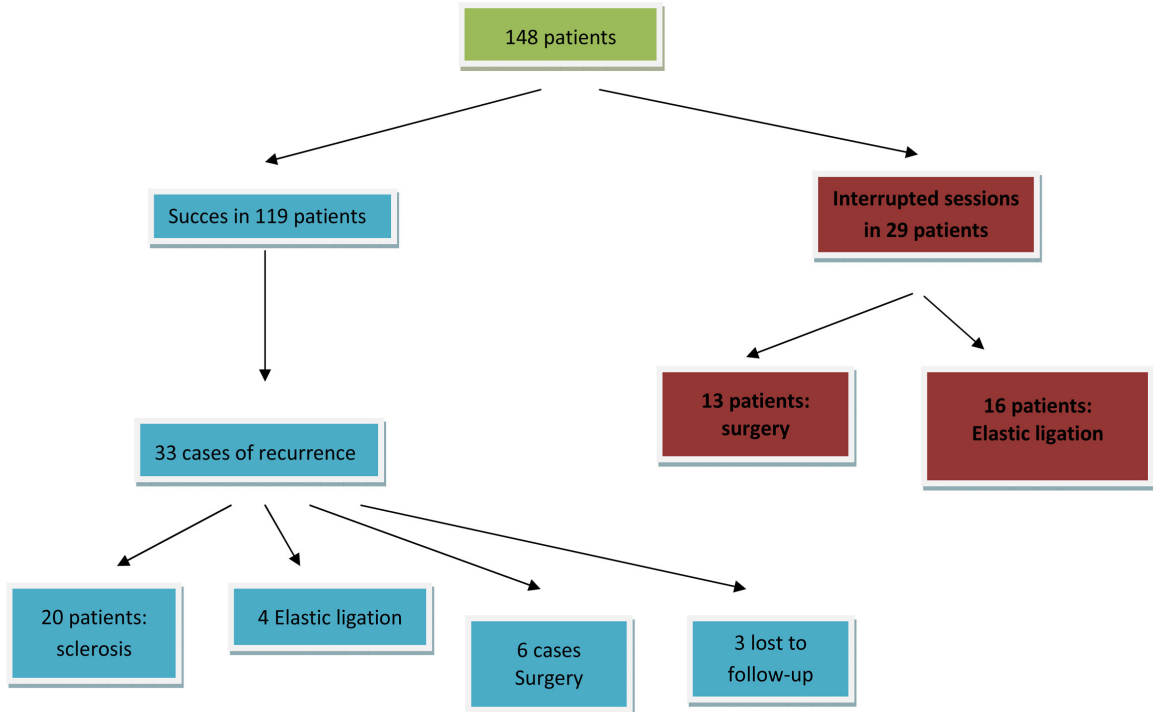

Figure 2. Flowchart of the enrolled patients. 
total, for grades 1 and 2 hemorrhoidal prolapse, the practitioner has the choice in a wide range of instrumental treatments and will choose the one of which he has the most expertise. In grade 3 hemorrhoidal prolapse, only elastic ligations proved effective in three quarters of cases. But if elastic ligation is the most effective technique in the long term, it generates secondary complications that are rare but can be serious [8].

In our series, initial success was found in 119 cases (80.4\%) after an average of 2.25 sessions $(1-4)$. The initial evolution was specified with a average follow-up of 3 months ( 1 - 13 months). The recurrence rate was $22.3 \%$ ( $\mathrm{N}: 33$ cases) after an average follow-up of 9.5 months ( 1 - 48 months). In the long term (over one year) and in the short term, the recidivism rate was $6 \%(\mathrm{~N}=9$ cases) and $16.2 \%$ $(\mathrm{n}=24)$ respectively. Among recurrent patients, the sclerosis protocol was repeated in 20 patients $(60.6 \%)$ with a success rate of $85 \%$.

Severe complications and sequelae are rare [4]: moderate pain or transient heaviness $(9 \%$ to $70 \%)$, minimal rectal bleeding ( $2 \%$ to $10 \%)$ and rare seepage [4] [9] [10]. Several cases of serious infectious complications have been reported and 3 cases of impotence, preceded by hemospermia or hematuria, have been reported after injection into the prostatic zone. According to these elements, prophylactic antibiotic treatment continued for 3 days can be advised (expert opinion) [2]. The prescription of an analgesic class 1 or 2 may be recommended, combined if necessary with a transit regulator [2]. In our series, 12 patients (8.1\%) had minor complications dominated by minimal spontaneous recursions.

\section{Conclusions}

Sclerotherapy is an effective technique for the treatment of symptomatic internal hemorrhoids, especially grades 1 and 2. It provides good short term results. It carries a risk of complications which often remain benign.

In our series, sclerotherapy of internal hemorrhoids resulted in an initial success of $80.4 \%$, with low complication rates $(8.1 \%)$ and an acceptable recurrence rate $(22.3 \%)$.

\section{Conflicts of Interest}

The authors declare no conflicts of interest regarding the publication of this paper.

\section{References}

[1] Coulom, P. (2009) Non Surgical Therapy of Hemorrhoids. Gastroentérologie Clinique et Biologique, 33, F20-F26.

[2] Higuero, T., Abramowitz, L., Staumont, G., et al. (2014) Clinical Practice Guidelines for the Treatment of Hemorrhoid Disease. Société Nationale Françoise de Colo-Proctologie (SNFCP), in Press.

[3] MacRae, H.M. and McLeod, R.S. (1995) Comparison of Hemorrhoidal Treatment Modalities. A Metaanalysis. Diseases of the Colon \& Rectum, 38, 687-694. https://doi.org/10.1007/BF02048023 
[4] Johanson, J.F. and Rimm, A. (1992) Optimal Nonsurgical Treatment of Hemorrhoids: A Comparative Analysis of Infrared Coagulation, Rubber Band Ligation, and Injection Sclerotherapy. The American Journal of Gastroenterology, 87, 1600-1606.

[5] Proktos.com injections sclérosantes.

[6] Bigard, M.A. (2004) Traitement médical endoscopique et chirurgical des maladies du tube digestif: Masson.

[7] Sim, A.J., Murie, J.A. and Mackusie, I. (1981) Comparison of Rubber Band Ligation and Sclerosant Injection for First- and Second-Degree Hemorrhoids. A Prospective Clinical Trial. Acta chirurgica Scandinavica, 147, 717-720.

[8] Tarrerias, A.L. and Guyot P. (2001) Pathologie hémorroïdaire. Digest, 2, 47 p.

[9] Walker, A.J., Leicester, R.J., Nicholls, R.J. and Mann, C.V. (1990) A Prospective Study of Infrared Coagulation, Injection and Rubber Band Ligation in the Treatment of Hemorrhoids. International Journal of Colorectal Disease, 5, 113-116. https://doi.org/10.1007/BF00298482

[10] Abramowitz, L., Sobhani, I., Benifla, J.L., et al. (2002) Anal Fissure and Thrombosed External Hemorrhoids before and after Delivery. Diseases of the Colon \& Rectum, 45, 650-655. https://doi.org/10.1007/s10350-004-6262-5 